\title{
CP3 STARS IN OPEN CLUSTERS
}

\author{
H.SCHNEIDER \\ Universitäts-Sternwarte, Geismarlandstr.11, D-3400 Göttingen, Germany
}

\begin{abstract}
Abt published in 1979 frequencies of peculiar stars in open clusters based on published classifications for 661 stars in 14 open clusters and associations. Among others he discussed an age dependence of the frequencies of CP3 stars and found them increasing with age. Furthermore, he suggested a possible age dependence of the rotational velocity of these stars.

But the sample he used was rather small: he found eight CP3 stars in 5 open clusters. In the mean time the number of cluster CP3 stars was more than double. Therefore, the findings of Abt shall be examined on the basis of the larger sample.
\end{abstract}

\section{INTRODUCTION}

From his sample Abt found eight CP3 out of 210 stars in 5 clusters. Another star (HR8473) is a member of the (doubtful) Ursa Major stream. It shows strong lines of both $\mathrm{Hg}$ and $\mathrm{Si}$, but no enhanced $\mathrm{Mn}$. It is certainly not a CP3 star and therefore not included in the discussion.

He divided the clusters in four age groups $\left(t=10^{5.7}, 10^{6.7}, 10^{7.4}\right.$ and $10^{8.3}$ years) to get more valid results and suggested an increasing of the frequencies with age, reaching the values for field stars at $t>10^{8}$ years. The latter value $(4.5 \%)$ was estimated from Osawa's sample (1965) or (5.8\%) taken from Wolff (priv. comm., cited in Abt), respectively. After $10^{8.8}$ years the hydrogen core is exhausted and no more CP3 stars appear.

\section{FREQUENCIES OF CP3 STARS IN OPEN CLUSTERS}

In the catalogue of Schneider (1981) 18 CP3 stars in nine open clusters out of 103 positive member of this peculiarity class are listed, yielding a percentage of $17.3 \%$ in total. These stars are listed in Table I. The age of the clusters, if not found in the literature, was estimated using the method of Mermillod (1981). The total number of stars in the same temperature range is given in column seven. In the cases where no MK classification could be found rough temperatures were estimated by means of published $U B V$ or $u v b y \mathrm{H} \beta$ data. Only stars up to $V=10^{m} .5$ have been considered (faintest CP 3 star in the catalogue). 
TABLE I CP3 stars in open clusters

\begin{tabular}{|c|c|c|c|c|c|c|c|}
\hline $\begin{array}{l}\text { cluster } \\
\text { ident. }\end{array}$ & ident. $^{\text {star }}$ & HD & $\begin{array}{c}v \cdot \sin i \\
{\left[\mathrm{~km} \mathrm{~s}^{-1}\right]}\end{array}$ & SB & $\begin{array}{c}\log t \\
\text { [years] }\end{array}$ & $\begin{array}{l}\text { B6-A } \\
\#_{\text {tot }}\end{array}$ & $\begin{array}{l}40 \\
\% C P 3\end{array}$ \\
\hline Ori I Ass & & 33647 & 28 & SB1 & 6.7 & 21 & 4.7 \\
\hline Sco I Ass & & $\begin{array}{l}144661 \\
144844\end{array}$ & $\begin{array}{l}40 \\
20\end{array}$ & $\begin{array}{c}\text { SB } \\
\text { SB2 }\end{array}$ & 6.7 & 40 & 5.0 \\
\hline IC4665 & $\begin{array}{l}\text { Kopff } 49 \\
\text { Kopff } 76 \\
\text { Kopff } 82\end{array}$ & $\begin{array}{l}161480 \\
161698 \\
161733\end{array}$ & $\begin{array}{l}25 \\
80 \\
40\end{array}$ & $\begin{array}{l}\text { SB1 } \\
\text { SB1 } \\
\text { SB1 }\end{array}$ & 7.4 & 19 & 15.8 \\
\hline IC4756 & $\begin{array}{l}\text { Kopff } 40 \\
\text { Kopff } 58 \\
\text { Kopff } 117\end{array}$ & $\begin{array}{l}171931 \\
172012 \\
172240\end{array}$ & - & - & 7.6 & 23 & 13.0 \\
\hline NGC2323 & Hoag 9 & - & - & - & 7.9 & 10 & 10.0 \\
\hline Pleiades & TS149 & 23950 & 80 & SB1 & 7.9 & 21 & 4.8 \\
\hline NGC2516 & $\begin{array}{l}\text { Cox } 60 \\
\text { Cox B } \\
\text { Cox b } \\
\text { Cox } 20 \\
\text { Cox } 23\end{array}$ & $\begin{array}{l}65949 \\
65950 \\
\\
66259 \\
66409\end{array}$ & $\begin{array}{l}20 \\
25 \\
40 \\
40\end{array}$ & $\begin{array}{l}\text { SB1 } \\
- \\
- \\
\text { SB1 } \\
\text { V2A }\end{array}$ & 8.1 & 49 & 10.2 \\
\hline NGC1039 & В 90 & 16693 & 25 & $\mathrm{~V} 2 \mathrm{~B}$ & 8.3 & 19 & 5.3 \\
\hline NGC2251 & Hoag 1 & 259954 & - & SB1 & 8.6 & 11 & $9.1^{\star}$ \\
\hline
\end{tabular}

* This star is certainly a blue straggler

The percentage of CP3 stars among the 213 cluster stars is $8.5 \%$. This value is larger than the one published by $\mathrm{Abt}$ and larger than the value for field stars from $\mathrm{Abt}(4.5 \%)$ or Wolff (5.8\%). Including the stars of those cluster where no CP3 star could be found the frequency will be reduced to $4.4 \%$. But on the other hand new investigations of the frequencies of CP stars (see Schneider's paper in this proceedings on "'Statistics of CP stars in a magnitude-limited sample: The Bright Star Catalogue") give a value of $9.6 \%$ for stars up to $V=6^{m} \cdot 0$. If this value is true the incidence of CP3 stars found in open clusters is slightly underestimated or normal.

Figure 1 shows the distribution vs. age. Abt frequencies $(0)$ and the ones found in this investigation ( $\bullet$ and $\bullet$ ) are marked. For the sake of completeness the single values of the whole sample are drawn in $(x)$, too. The different frequencies for field CP3 stars are indicated by arrows. 


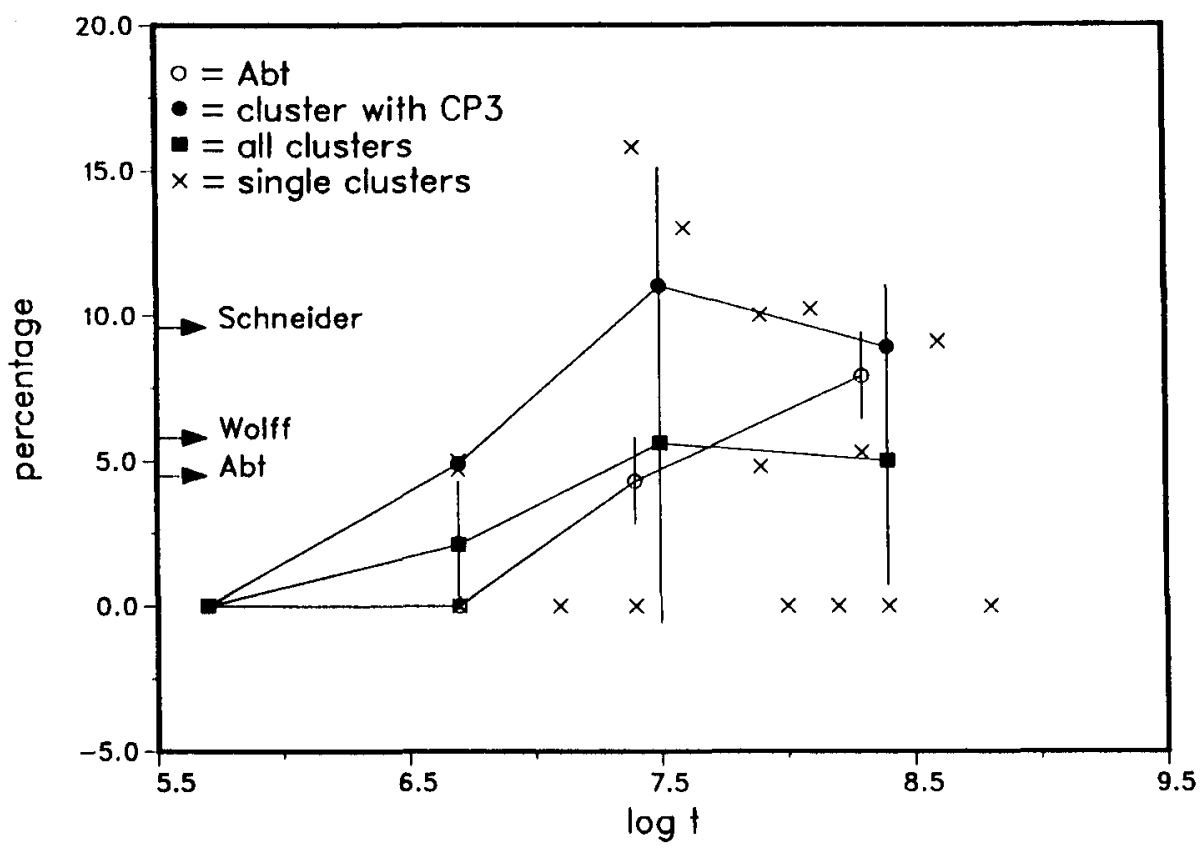

Fig. 1. Distribution of frequencies vs. age of CP3 stars in open clusters.

Because of different observational material the age groups have been modified a little: $10^{7.5}$ instead of $10^{7.4}$ and $10^{8.4}$ instead of $10^{8.3}$ years. Also, the ages for some of the individual clusters differ slightly from those given by Abt, but this does not influence the averaged values. In Table 2 the results are tabulatèd.

TABLE II Age dependence of CP3 stars

\begin{tabular}{ccccccccccc}
\hline age group & \multicolumn{3}{c}{$\begin{array}{c}\text { clusters (9) with } \\
\text { CP3 stars }\end{array}$} & \multicolumn{3}{c}{ all clusters (16) } & \multicolumn{3}{c}{ Abt's sample (5) } \\
[years] & no. & freq. & $\sigma$ & no. & freq. & $\sigma$ & no. & freq. & $\sigma$ \\
\hline $10^{5.7}$ & $0 / 0$ & $0.0 \%$ & & $0 / 0$ & $0.0 \%$ & & $0 / 7$ & $0.0 \%$ & \\
$10^{6.7}$ & $3 / 61$ & $4.9 \%$ & 0.2 & $3 / 141$ & $2.1 \%$ & 2.2 & $0 / 80$ & $0.0 \%$ & \\
$10^{7.4}$ & & & & & & & $2 / 47$ & $4.3 \%$ & 1.5 \\
$10^{7.5}$ & $8 / 73$ & $11.0 \%$ & 4.1 & $8 / 143$ & $5.6 \%$ & 6.2 & & & \\
$10^{8.3}$ & & & & & & & $6 / 76$ & $7.9 \%$ & 1.5 \\
$10^{8.4}$ & $7 / 79$ & $8.9 \%$ & 2.1 & $7 / 141$ & $5.0 \%$ & 4.3 & & & \\
\hline \hline
\end{tabular}

The scattering of the frequencies of CP3 stars in the individual clusters is large and, therefore, the rms of the averaged data. This reflects the fact that some clusters show a large incidence of CP3 stars while others do not show even 
one. If one takes only the clusters with CP3 stars it seems that an age dependence exists. But I doubt that this is right but rather a selection effect. Including the clusters with no CP3 star there is no evidence for such dependence. The latter statement is supported by the preliminary results for CP2 stars of the cluster program in the framework of the European Working Group on CP Stars.

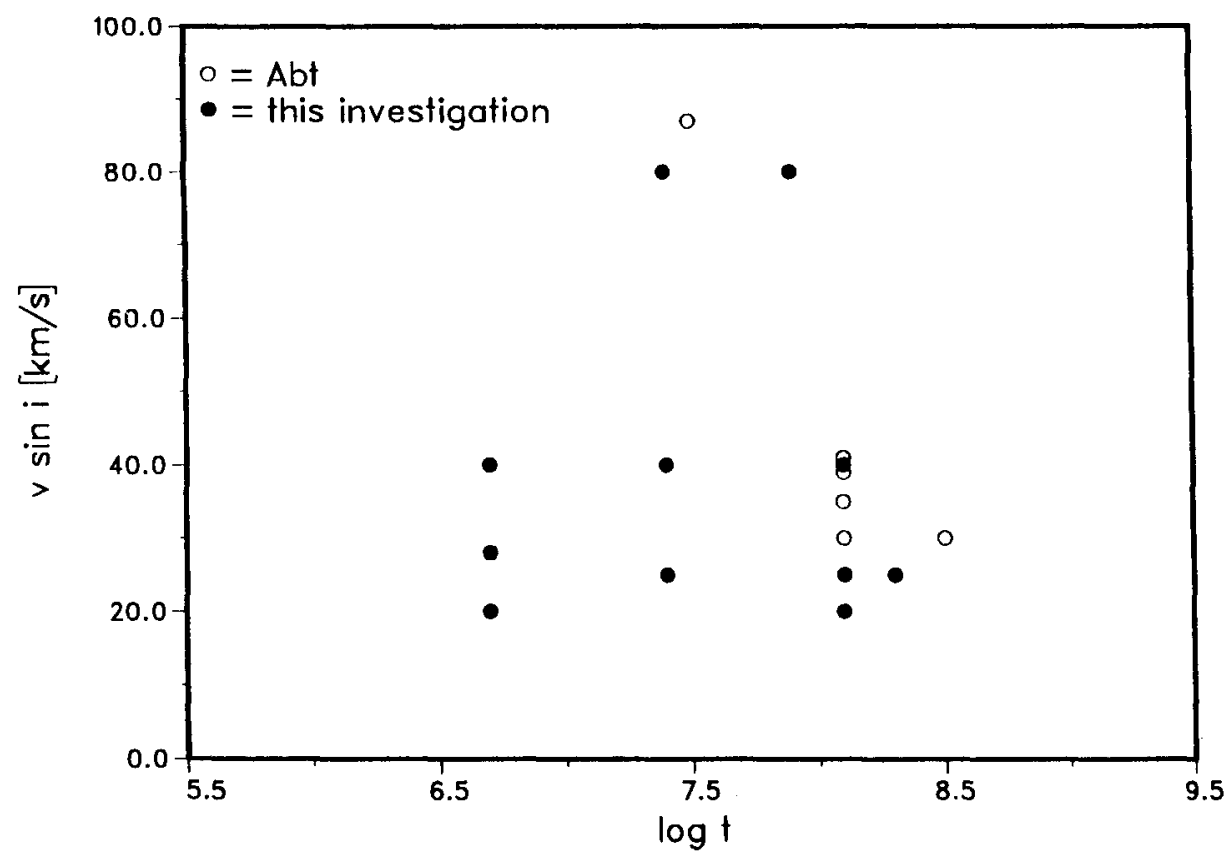

Fig. 2. Distribution of $v \cdot \sin i$ vs. age of CP3 stars in open clusters.

\section{ROTATIONAL VELOCITIES OF CP3 STARS IN OPEN CLUSTERS}

Another suggestion of $\mathrm{Abt}$, the decreasing of $v \cdot \sin i$ with age, shall also be examined. Although only weakly indicated he interpreted this as a braking of the rotation on the main sequence. But again, his suggestion is based only on eight stars in five clusters. In the mean time more and better $v \cdot \sin i$ values are available (for 12 stars). Figure 2 shows the distribution of the stars $(\bullet)$. Abt's values are also indicated (o). It is evident that no age dependence of $v \cdot \sin i$ exists.

Two stars show a distinctly larger values $\left(v \cdot \sin i=80 \mathrm{~km} \mathrm{~s}^{-1}\right)$. But one of them (HD23950) shows indication of an unresolved SB2 system which means that the published $v \cdot \sin i$ value is too large.

The mean value of the sample (12 stars) is

$$
\langle v \cdot \sin i\rangle_{\text {clusters }}=38.6 \pm 20.0 \mathrm{~km} \mathrm{~s}^{-1} .
$$

Neglecting the the two larger values yields 


$$
\langle v \cdot \sin i\rangle_{\text {clusters }}=30.3 \pm 8.2 \mathrm{~km} \mathrm{~s}^{-1} \text {. }
$$

This result is comparable with the mean value of $29.6 \mathrm{~km} \mathrm{~s}^{-1}$ for all CP 3 stars.

\section{CONCLUSIONS}

CP3 stars are hard to detect with moderate dispersion. High resolution spectroscopy of faint stars, e.g. for most of the cluster stars, is very time consuming and, therefore, the data base is biased towards accidential detections. Nevertheless, 18 stars CP3 stars in nine clusters are known. An age dependence suggested by $\mathrm{Abt}$ is only marginal indicated but certainly not significant. Another suggestion, the age dependence of the rotational velocity, could not be confirmed.

The behaviour of CP3 stars in open clusters indicates that the CP3 phenomena should exit before the stars reach or come close to the main sequence. While staying there no or only marginal evolution takes place.

Most of the stars in Table 1, so far as spectroscopic data exist, are spectroscopic binaries or members of visual binary systems. This supports the opinion that all CP3 stars are binaries.

\section{REFERENCES}

Abt,H.A., 1979, Ap. J., 230,485

Mermillod, J.C., 1981, Astr. Ap. Suppl., 44,467

Osawa,K. 1965: Ann. Tokyo Astron. Obs. Ser. II, 9,121

Renson,P., 1992, Inform. Bull. CDS, 40,97

Schneider,H., 1981, Astr. Ap. Suppl., 44,137 